\title{
Variations
}

Variations Revue internationale de théorie critique

15 | 2011

La haine

\section{Éloge de la raison}

Les lumières de Thilo Sarrazin

\section{Hans Horch}

Traducteur : Pascale Jahns-Petit

\section{OpenEdition}

\section{Journals}

Édition électronique

URL : http://journals.openedition.org/variations/86

DOI : 10.4000/variations.86

ISSN : 1968-3960

\section{Éditeur}

Les amis de Variations

\section{Édition imprimée}

Date de publication : 1 mars 2011

\section{Référence électronique}

Hans Horch, «Éloge de la raison », Variations [En ligne], 15 | 2011, mis en ligne le 01 février 2012, consulté le 21 avril 2019. URL : http://journals.openedition.org/variations/86 ; DOI : 10.4000/ variations.86 


\section{Hans Horch}

Eloge de la raison

\section{Les lumières de Thilo Sarrazin}

Au Parlement allemand, il n'y a pas un seul député qui soit membre d'un parti féru de diatribes xénophobes. Pour être représenté, il faudrait déjà qu'un tel parti soit en mesure d'atteindre la barre des cinq pour cent de voix aux élections.

Car les Allemands ont appris une chose : l'extrémisme politique n'est pas convenable. Aussi, les émotions sont à bannir du discours politique. L'opinion devrait pouvoir se former à partir de l'observation lucide des faits, suivie de l'analyse claire, logique et scientifique de ces derniers - et ce justement lorsqu'il s'agit d'un sujet aussi passionné que celui de l'immigration. Ainsi l'opinion publique allemande demeure-t-elle épargnée des Wilders, Strache, Blocher, Le Pen ou encore d'une Kjaersgaard. Cette grâce faite à l'Allemagne bénéfice également à Thilo Sarrazin. Celui-ci, en effet, s'entend à merveille pour justifier de façon purement rationnelle ses verdicts implacables au sujet des immigrés et des couches sociales défavorisées, en s'appuyant sur des chiffres et des données ainsi que sur une connaissance poussée des références spécialisées, et en se servant d'une science aussi exacte que la biologie pour étayer le point de vue d'une économie politique bien trop souvent spéculative. Alors que dans les pays voisins, force est de reconnaître qu'on exhorte aux passions et à la haine, Thilo Sarrazin élève la voix du bon sens en Allemagne devant un public qui n'attend que cela. Sarrazin se senti incompris à la suite d'une interview parue à l'automne 2009 dans la revue «Lettre international », où il déclara «Si les 1,3 milliard de Chinois sont aussi intelligents, mais plus travailleurs et bientôt plus qualifiés que les Allemands, alors que ces derniers adoptent de plus 
en plus la mentalité turque, on va avoir de gros problèmes » (« Wenn 1,3 Milliarden Chinesen genauso intelligent sind wie die Deutschen, aber fleißiger und in absehbarer Zeit besser ausgebildet, während wir Deutschen immer mehr eine türkische Mentalität annehmen, bekommen wir ein größeres Problem. »)

En réaction aux critiques, il sacrifia gracieusement le peu de temps libre que lui laissait le directoire de la Bundesbank et rédigea une étude à prétention hautement scientifique qu'il intitula de manière aussi sobre que cela lui fut possible : « Deutschland schafft sich ab » ou « L'Allemagne court à sa perte. »

Dans son entretien, partant du fait certes vérifiable, selon lequel les personnes peu qualifiées sont touchées plus durement par le chômage de longue durée, il en déduit à sa manière que le manque de qualification serait la seule origine du chômage. Toujours selon lui, la réussite économique de chaque individu dépendrait du niveau de formation, tout comme la réussite d'une économie politique dépendrait de l'intelligence moyenne de sa population. Il en serait de même de la répartition géographique des forces économiques : car les investissements iraient là où se trouverait le meilleur capital de ressources humaines et, douterait-on encore de l'intelligence supérieure de Thilo Sarrazin, nous en aurions pour preuve son implacable analyse.

De même, les grandes entreprises industrielles ainsi que le tertiaire se trouveraient à Cologne, Munich, Hambourg ou encore à Francfort, en raison de la concentration des meilleurs managers, des développeurs les plus renommés, des plus fameux avocats, de consultants, d'ingénieurs, de scientifiques, etc. La situation économique désastreuse de Berlin s'expliquerait, pour sa part, du fait qu'un trop petit nombre de piliers économiques contre un trop grand nombre de personnes improductives, et donc peu intelligentes, y vivraient. Non contente d'être très large, la 
couche sociale inférieure, en outre, se reproduirait biologiquement plus rapidement que la couche supérieure, celle des performants, de cette population infatigable qui ne serait plus en mesure de suivre le rythme, et ce pour une raison bien simple : cette couche sociale se comporte en grande partie d'Arabes et de Turcs certes économiquement improductifs, mais se prévalant d'un taux de reproduction démographique élevé. Toujours selon Thilo Sarrazin, invoquant les taux de chômage qui frappent ces groupes, leur comportement parasitaire envers leur environnement serait irrévocable puisque inné.

La couche sociale inférieure se développant plus vite que celle des qualifiés et des performants, le degré moyen d'intelligence de la population risquerait de baisser de génération en génération, dans l'imaginaire de Sarrazin. Que faire alors ? Il offre une seule et logique réponse : un échange de populations serait nécessaire ! Il faudrait freiner la croissance de la couche inférieure, voire même l'inverser. Le retrait des allocations ainsi que l'interdiction des mariages empêcheraient les immigrés improductifs de se reproduire et les inciteraient à quitter le pays. A eux de « cesser de se reproduire » ou, pour citer encore une réponse de T. Sarrazin, de « disparaître. » En revanche, les familles des peuples intelligents et travailleurs seraient invitées à immigrer en Allemagne, plus spécialement les Juifs d'Europe de l'Est, dont le quotient intellectuel dépasserait de $15 \%$ la moyenne allemande, chiffre qu'il croit provenir d'exacts calculs scientifiques. Non, Sarrazin ne se présente pas comme étant xénophobe, ni d'extrême droite. Il se contente de penser en termes économiques et biologiques. D'ailleurs, l'appel à la haine et au pogrom relèverait pour lui d'un comportement typique de la couche sociale inférieure. Il vise pour sa part des mesures étatiques, prises dans un cadre législatif.

Sachant que Sarrazin prône également des mesures relatives à l'éducation, comment à vrai dire ne pas être quelque peu déconcerté ? En effet, si lors de l'interview au cours duquel il prônait 
l'échange de population, il avait certes déclaré inutile toute prise en charge éducative d'éléments condamnés par nature à l'improductivité économique, il n'en demeure pas moins que dans son livre, il exige leur pure et simple scolarisation. Une fois la façon de penser de Sarrazin bien comprise, cette contradiction cesse d'être apparente et se révèle rusée : les écoles dissuaderaient en toute évidence les doctes familles susceptibles d'immigrer en Allemagne du fait qu'elles accueilleraient des enfants des couches inférieures.

Nonobstant le respect que nous devons à l'argumentation de Sarrazin, sa théorie sur la baisse de la moyenne d'intelligence se heurte néanmoins à un fait significatif, à savoir le succès de son propre livre. Bien que l'auteur exige du lecteur un niveau intellectuel élevé, deux millions d'exemplaires s'étaient déjà vendus fin 2010. Les lecteurs sondés affirment retrouver chez l'auteur, sous une forme scientifique, ce qu'ils penseraient spontanément. Qu'à cela ne tienne ! Comment ne pas en déduire que les personnes intelligentes ne s'opposeraient pas au nombre croissant de sots? Que tout individu serait en mesure de promouvoir son intelligence ? Ne serait-ce pas la preuve ultime que les citoyens instruits auraient enfin appris à ne plus expliquer sociologiquement les faits biologiques manifestes ? Qu'ils auraient admis que la réussite économique par la performance implique une sélection fondée sur l'utilité ? Que celui qui mise sur une régulation publique du marché du travail plutôt que d'être son propre employeur doit disparaître ? Qu'il s'agirait de barrer la route à ceux-là mêmes qui entraveraient la concurrence et la course à la productivité en raison de la paresse récompensée par des mesures de politique sociale, et la performance individuelle punie par des impôts collectifs ? Ceux-là ne poignarderaient-ils pas dans le dos les actifs, auxquels ils sont redevables de leur bien-être ? Que la nation risque de perdre la lutte qu'elle mène à l'échelle mondiale pour sa survie économique en tolérant trop d'existences inaptes dans ses rangs ? Que par conséquent, ce qui semble humain nuit à l'homme? 
On le sait, de par le passé, les érudits en Allemagne étaient enclins à interpréter la société et la politique avec des constructions métaphysiques ou à corriger le monde en fonction de leur moralité supérieure. Aujourd'hui, c'est fini. Les lecteurs allemands de Sarrazin sont revenus à la raison froide. Ils ont finalement reconnu et approuvé la réalité de la société dans laquelle ils vivent. Voilà ce qu'ils expriment en plébiscitant ce livre et son auteur. Ce dernier, quant à lui, a fait preuve d'une grande intuition pour déceler ce qui agite le centre de la société et l'a projeté avec brio sur la scène publique. Un acte digne des Lumières s'il en est!

Hans Horch

Traduit de l'allemand par Pascale Jahns-Petit 